\title{
Kinetic Limitations on the Use of Redundancy in Robotic Manipulators
}

\author{
Anthony A. Maciejewski, Member, IEEE
}

\begin{abstract}
The kinematic specification of motion for redundant manipulators has relied primarily on a formulation that decomposes joint velocity solutions into a pseudoinverse component and a homogeneous solution component. While such a formulation is conceptually appealing, since it treats the redundant degrees of freedom as independent from those required to maintain a desired end effector trajectory, it has been shown to be physically inaccurate when applied to the kinetic behavior of redundant manipulators. In this work, the kinetic behavior of the homogeneous solution component is analyzed in order to specify realistic limitations on the use of redundancy. It is shown that the equations that govern these limitations are related to the conditions for guaranteeing stability of the local torque minimization formulation.
\end{abstract}

\section{INTRODUCTION}

THE vast majority of efforts to utilize redundancy in robotic manipulators have been focused on the resolution of redundancy at the kinematic level. The kinematics of manipulators is frequently represented by

$$
\dot{x}=J \dot{\theta}
$$

where $\dot{x}$ is an $m$-dimensional vector specifying the end effector velocity, $\dot{\theta}$ is an $n$-dimensional vector denoting the joint velocities, and $J$ is the $m$ by $n$ Jacobian matrix. For redundant manipulators $n>m$ so that the general solution to (1) is typically presented in the form

$$
\dot{\theta}=J^{+} \dot{x}+\left(I-J^{+} J\right) \dot{\phi}
$$

where ${ }^{+}$denotes the pseudoinverse, $\left(I-J^{+} J\right)$ is a projection operator onto the null space of $J$, and $\dot{\phi}$ is an arbitrary vector in $\dot{\theta}$ space. The second term in (2) is the homogeneous solution to (1) since it results in no end effector velocity and will be denoted here by $\dot{\theta}_{H}$. This homogeneous solution is frequently used to optimize some secondary criterion under the constraint of the specified end effector velocity by choosing $\dot{\phi}$ to be the gradient of some function of $\theta$ [13]. Alternative formulations for instantaneously optimizing a secondary criterion by augmenting the Jacobian matrix have also been presented [1], [5]. Some of the secondary criteria that have been applied include joint range availability [12], singularity avoidance [16], [18], various measures of dexterity [6], [7], [10], [19], [20], and obstacle avoidance [14], [18]. The homogeneous solution can also be used to optimize secondary criteria defined in Cartesian space, either to impose a priority to the manipulation variables [17] or to avoid obstacles [14], by using

$$
\dot{\phi}=\left[J_{2}\left(I-J^{+} J\right)\right]^{+}\left(\dot{x}_{2}-J_{2} J^{+} \dot{x}\right)
$$

Manuscript received May 25, 1989; revised March 6, 1990. This work was supported by the NEC under a Faculty Fellowship. This paper was presented at the 1989 IEEE International Conference on Robotics and Automation, Scottsdale, AZ, May 14-19, 1989.

The author is with the School of Electrical Engineering, Purdue University, West Lafayette, IN 47907.

IEEE Log Number 9042517. where the subscript 2 refers to the secondary criterion. The overall solution is then given by substituting (3) into (2) to obtain

$$
\dot{\theta}=J^{+} \dot{x}+\left[J_{2}\left(I-J^{+} J\right)\right]^{+}\left(\dot{x}_{2}-J_{2} J^{+} \dot{x}\right)
$$

which has been simplified by taking advantage of the fact that the projection operator is Hermetian and idempotent [14].

In all of the above techniques, the specified end effector trajectory is the implicit primary criterion. Unfortunately, the specification of an arbitrary homogeneous joint velocity may result in unrealistic demands on manipulator performance. These difficulties were first illustrated in [11] where the dynamic performance of a redundant manipulator showed significant end effector tracking errors when a secondary criterion was imposed. A more dramatic difficulty with using homogeneous solutions is the instability illustrated in [8] when redundancy is resolved at the acceleration level to instantaneously minimize joint torque. In this case, the joint acceleration is related to the end effector acceleration by differentiating (1) to obtain

$$
\ddot{x}=J \ddot{\theta}+\dot{J} \dot{\theta}
$$

where once again the general solution is expressed in the form

$$
\ddot{\theta}=J^{+}(\ddot{x}-\dot{J} \dot{\theta})+\left(I-J^{+} J\right) \ddot{\phi} .
$$

The dynamic equations of a manipulator can be written in closed form as

$$
\tau=H \ddot{\theta}+c+g
$$

where $\tau$ is the vector of joint torques, $H$ is the inertia matrix, $c$ is a vector of torques due to Coriolis and centrifugal effects, and $g$ is the gravity vector. The elements of the vector $c$ can be written in quadratic form, so that

$$
c_{i}=\dot{\theta}^{T} C_{i} \dot{\theta}
$$

where the $C_{i}$ are matrices of Coriolis and centrifugal coefficients. If $\tilde{\tau}$ is used to denote the torque due to the minimum norm acceleration, then $\tilde{\tau}$ can be obtained by substituting the first term of (6), i.e., the pseudoinverse solution, into (7), which results in

$$
\tilde{\tau}=H J^{+}(\ddot{x}-\dot{j} \dot{\theta})+c+g .
$$

It has been shown [8] that one can sacrifice the minimum norm acceleration in order to locally minimize the norm of the torque by using a solution of the form

$$
\ddot{\theta}=J^{+}(\ddot{x}-\dot{J} \dot{\theta})-\left[H\left(I-J^{+} J\right)\right]^{+} \tilde{\tau}
$$

which uses a homogeneous acceleration term in a manner analogous to that of (4). The value of this minimum torque can be 
obtained by substituting (10) into (7) and then using (9) to obtain

$$
\tau_{\min }=\tilde{\tau}-H\left[H\left(I-J^{+} J\right)\right]^{+} \tilde{\tau} .
$$

Unfortunately, because this is only a local minimization technique, it has been shown that the joint acceleration given by (10) can induce large joint velocities that may require physically unrealizable joint torques in order to maintain the desired end effector trajectory.

In this work, in order to place realistic limitations on the use of redundancy, the kinetic effects of a homogeneous solution will be analyzed. It will be shown that an arbitrary homogeneous solution cannot be used without potentially affecting the primary constraint of a desired end effector trajectory. In addition, conditions for identifying the instability of the torque minimization technique will be presented. It will be shown that these conditions are only a function of a manipulator's configuration and thus can be used to determine desirable regions of operation.

\section{Kinetic Effects of A Homogeneous SOlution}

In order to explicitly consider the kinetic effects of a homogeneous velocity, only the case where $\ddot{x}=\dot{x}=0$, that is, the desired end effector trajectory requires the hand to remain stationary at a given position and orientation, will be considered. This case occurs in practice whenever a reconfiguration of the manipulator is required to avoid a moving obstacle or as the result of a residual homogeneous velocity after the desired end effector trajectory is completed. Under these conditions, there will in general be an acceleration at the end effector due to the rate of change of $J$ as given by (5). The resulting joint angle acceleration required to maintain the desired configuration of the end effector is given by (6), which if one assumes that there is to be no acceleration along the homogeneous solution results in

$$
\ddot{\theta}=-J^{+} \dot{j} \dot{\theta}_{H} .
$$

If the discussion is restricted to manipulators with a single degree of redundancy, then the acceleration given by (12) will result in a constant magnitude of homogeneous velocity which will trace a curve in joint space that corresponds to all the possible manipulator configurations that can be reached without moving the end effector. The joint acceleration given by (12) has a simple physical interpretation in that it is inversely related to the radius of curvature of this homogeneous solution space curve. In particular, if the radius of curvature is denoted by $\rho$, then (12) can be written as

$$
\ddot{\theta}=-\frac{\left\|\dot{\theta}_{H}\right\|^{2}}{\rho} \hat{r}
$$

where $\hat{r}$ is a unit vector directed from the homogeneous solution curve toward the center of curvature. The two important points to note about this acceleration are that, first, its magnitude is proportional to the square of the magnitude of the homogeneous joint velocity, and second, that its direction is independent of not only the magnitude of $\dot{\theta}_{H}$ but also of the direction of $\dot{\theta}_{H}$ around the homogeneous solution curve. Therefore, the direction of the joint acceleration required to maintain the desired end effector trajectory is a function of only the manipulator configuration.

The torque required to maintain a constant homogeneous joint velocity is given by (9), which when one includes the constraint that the end effector remain stationary, i.e., $\ddot{x}=0$, results in

$$
\tilde{\tau}=-H J^{+} \dot{j} \dot{\theta}+c+g .
$$

If one neglects the velocity-independent gravity term and rewrites the joint angle acceleration term using the equality of (12) and (13), the following equation results:

$$
\tilde{\tau}=-H \frac{\left\|\dot{\theta}_{H}\right\|^{2}}{\rho} \hat{r}+c .
$$

The constant representing the magnitude of the homogeneous velocity squared can now be factored from both of the remaining terms, which results in

$$
\tilde{\tau}=\left\|\dot{\theta}_{H}\right\|^{2}\left[\frac{-H \hat{r}}{\rho}+c^{\prime}\right]
$$

where the elements of $c^{\prime}$ are given by

$$
c_{i}^{\prime}=v_{n}^{T} C_{i} v_{n}
$$

where $v_{n}$ is a unit vector along $\dot{\theta}_{H}$. In the case of a single degree of redundancy, $v_{n}$ corresponds to the $n$th output singular vector specifying the null space of $J$. Clearly, the torque given by (16) must be physically achievable in order to maintain the desired end effector trajectory. Now consider the case where an acceleration along the homogeneous solution is allowed. Such an acceleration, denoted by $\ddot{\theta}_{H}$ and given by the second term in (6), will affect the torque requirements in two ways: directly, through the inertial torque required to achieve the acceleration, and indirectly, through the increase or decrease of the homogeneous velocity. When using the instantaneous torque minimization formulation, the direct effect of $\ddot{\theta}_{H}$ is used to decrease the torque requirements by applying the acceleration given by (10). In this case the homogeneous acceleration term, which is represented by the second term in (10), can be obtained by substituting (16) for $\tilde{\tau}$, which results in

$$
\ddot{\theta}_{H}=\left\|\dot{\theta}_{H}\right\|^{2}\left[H\left(I-J^{+} J\right)\right]^{+} t
$$

where

$$
t=\left[\frac{H \hat{r}}{\rho}-c^{\prime}\right] .
$$

While this homogeneous acceleration term will minimize the instantaneous torque requirement, it tells us nothing about the indirect effect on future torque requirements. In order to obtain this information one must look at the direction of the homogeneous acceleration relative to the homogeneous velocity. Mathematically, if

$$
v_{n}^{T} \ddot{\theta}_{H}>0
$$

then the homogeneous acceleration term will increase the magnitude of the homogeneous velocity and subsequently increase the torque requirements. This, in effect, amounts to a positive feedback system and results in the instability of local torque minimization noted in [8]. Note that for systems with a single degree of redundancy the angle between $v_{n}$ and $\ddot{\theta}_{H}$ in (20) will be either $0^{\circ}$ or $180^{\circ}$.

In order to guarantee global stability when using the local torque minimization formulation, the homogeneous acceleration must not be applied when (20) is true. It is possible to identify regions of stability and instability for this formulation by evaluating the conditions for which (20) holds. Substituting (18) into the left-hand side of (20) results in

$$
v_{n}^{T} \ddot{\theta}_{H}=v_{n}^{T}\left\|\dot{\theta}_{H}\right\|^{2}\left[H\left(I-J^{+} J\right)\right]^{+} t .
$$


It is easy to show (see the Appendix) that

$$
\left[H\left(I-J^{+} J\right)\right]^{+}=\frac{v_{n} v_{n}^{T} H}{v_{n}^{T} H^{2} v_{n}}
$$

so that $(21)$ becomes

$$
v_{n}^{T} \ddot{\theta}_{H}=\left\|\dot{\theta}_{H}\right\|^{2} \frac{v_{n}^{T} H}{v_{n}^{T} H^{2} v_{n}} t .
$$

Since only the sign of $v_{n}^{T} \ddot{\theta}_{H}$ is of concern, (23) can be simplified to

$$
\operatorname{sign}\left(v_{n}^{T} \ddot{\theta}_{H}\right)=\operatorname{sign}\left(v_{n}^{T} H t\right)
$$

since $H$ is positive definite. There are two important observations concerning (24) that should be pointed out. First, the magnitude of the homogeneous joint velocity does not in any way affect the sign of $v_{n}^{T} \ddot{\theta}_{H}$. Second, the vector $H t$ is independent of the direction of the homogeneous velocity, being solely a function of the manipulator configuration. As a result of the second point, it is possible to determine regions of operation for which the local torque minimization method is inherently stable or unstable. The following section will present a specific example.

In addition to providing conditions for the stability of the instantaneous torque minimization formulation, the above discussion also pertains to the use of homogeneous solutions for realizing other secondary criteria. The torque requirements of a homogeneous velocity given by (16) along with the condition expressed by (20) identify when and to what degree a desired homogeneous joint velocity solution can be induced without resulting in unrealistic torque requirements. In particular, if the torque required to maintain a desired homogeneous velocity is approaching its physical limitation, (20) can be used to determine whether the magnitude of the homogeneous velocity should be reduced immediately or whether the manipulator should wait for a configuration where both the torque and velocity can be reduced simultaneously.

\section{A Two-Dimensional Example}

The issues presented in the previous section will be illustrated for the planar three-degree-of-freedom manipulator depicted in Fig. 1. The links are all identical and are modeled as thin uniform rods with lengths of $1 \mathrm{~m}$ and masses of $10 \mathrm{~kg}$. The homogeneous solution curves for this manipulator, which have been previously presented [3], [9], are shown in Fig. 2. These curves are plotted for end effector positions ranging from $x=$ $1.01 \mathrm{~m}$ for the outermost curve to $x=3.00 \mathrm{~m}$, which corresponds to the point at the origin. These curves are related to the homogeneous joint velocity $\dot{\theta}_{H}$ in that $\dot{\theta}_{H}$ is tangent to the curve corresponding to the current end effector position. These curves are generated by selecting the desired end effector position, specifying a homogeneous joint velocity of $1 \mathrm{rad} / \mathrm{s}$, and then applying the acceleration given by (12). An alternative technique for generating these curves is presented in [10]. An important observation concerning these curves is the wide variation in the radius of curvature. This is particularly noticeable for the homogeneous solution curve that goes through the three internal singular configurations labeled $S_{1}$ to $S_{3}$. Near these singular configurations the radius of curvature approaches zero, resulting in accelerations (and torques) that approach infinity in order to maintain a constant end effector position. The other sections of this curve, however, are nearly linear and thus require virtually

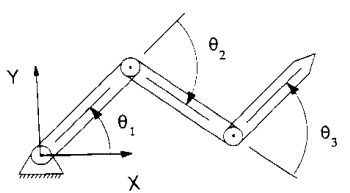

Fig. 1. Geometry of the planar three-link manipulator used in the examples All three link lengths are equal to $1 \mathrm{~m}$.

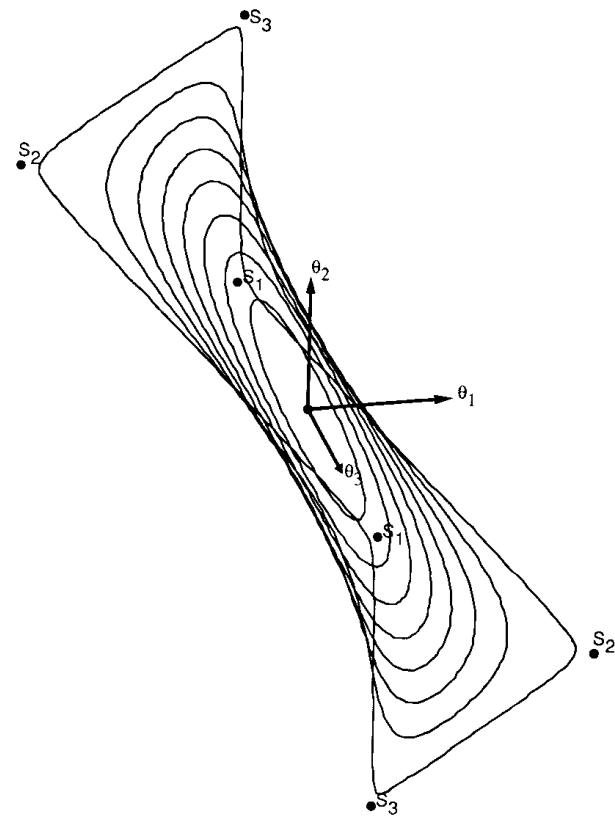

Fig. 2. A parallel projection of the homogeneous solution curves for the manipulator in Fig. 1 plotted in 3D $\theta$ space. The curves are plotted for end effector positions ranging from $x=1.01 \mathrm{~m}$ for the outermost curve to $x=3.00 \mathrm{~m}$, the full reach of the manipulator for which the curve is simply the point at the origin, at a spacing of $0.25-\mathrm{m}$ intervals. In all cases $y=0$. The three different internal singular configurations that occur at a distance of one link length from the base are shown at points $S_{1}, S_{2}$, and $S_{3}$. The axes in this figure are all of length $90^{\circ}$.

no acceleration in order to maintain the desired end effector position. Another view of these homogeneous solution curves is presented in Fig. 3 where the viewing direction is along the vector $\theta^{T}=\left[\begin{array}{lll}3 & 2 & 1\end{array}\right]$. This view is chosen because it tends to more accurately reflect the shape of these curves, particularly at reaches farther from the base, since these curves tend to lie in a plane with a surface normal of $\left[\begin{array}{lll}3 & 2 & 1\end{array}\right]$. This orientation is due to using equal link lengths that results in a $3: 2: 1$ ratio in the columns of $J$ when the manipulator is fully extended. A further discussion on some of the properties of these curves can be found in [4], [15].

In Fig. 4 are shown plots of the acceleration, inertial torque, and total torque required to maintain a homogeneous velocity of $1 \mathrm{rad} / \mathrm{s}$ when the end effector is commanded to be at a position $2.00 \mathrm{~m}$ from the base. Since the norm of the joint velocity is unity, the norm of the joint acceleration is also equal to the inverse of the radius of curvature of the homogeneous solution curve. The maximum and minimum accelerations denoted $A$ to $D$ can be shown to correspond with the maximum and minimum radiuses of curvature for the curve in Fig. 3. From these plots it is clear that while the inertial torques due to the radius of 


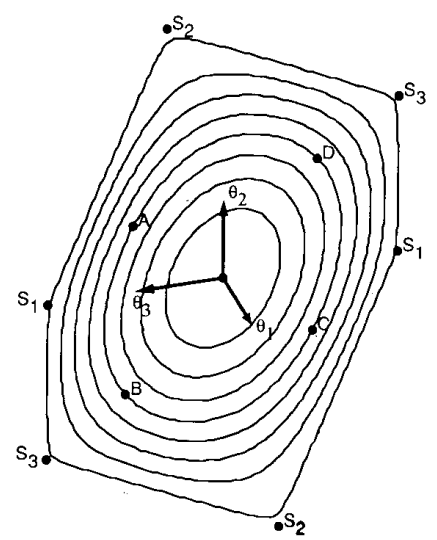

Fig. 3. Another view of the homogeneous solution curves from Fig. 2. The viewing direction is along the vector $\left[\begin{array}{lll}3 & 2 & 1\end{array}\right]$, which is the normal to the plane in which most of these curves approximately lie. The four points $A$, $B, C$, and $D$ correspond to the points of minimum and maximum radius of curvature for an end effector position of $x=2.00 \mathrm{~m}$.
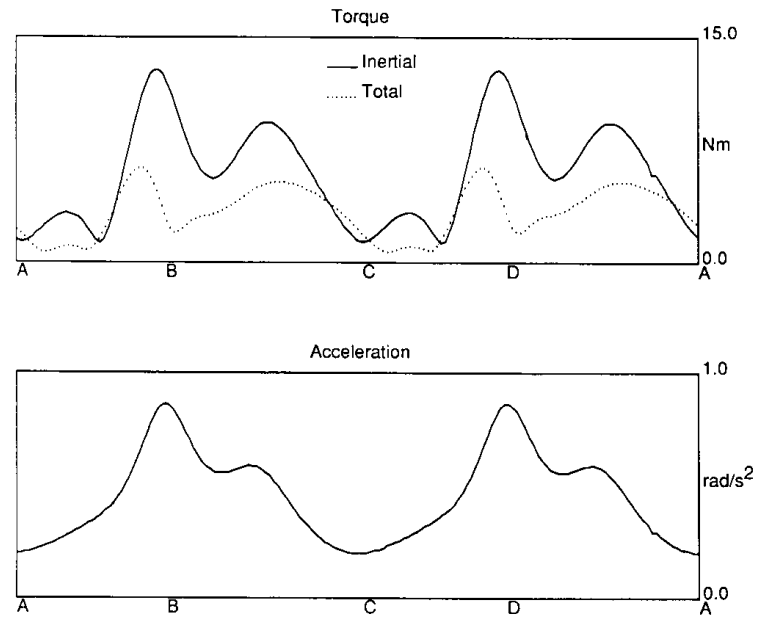

Fig. 4. Graphs of the acceleration, inertial torque, and total torque required to maintain a homogeneous velocity of $1 \mathrm{rad} / \mathrm{s}$ around the homogeneous solution curve for $x=2.00 \mathrm{~m}$.

curvature are the dominant characteristic in determining the overall torque requirements, the Coriolis and centripetal torques do play a significant role, typically mediating the effect of the inertial torques, and therefore cannot be ignored. It is important to note once again that the total torque required to maintain the homogeneous velocity, $\tilde{\tau}$ given by (16), is independent of the direction of that velocity. In other words, in terms of Fig. 3, $\tilde{\tau}$ is independent of whether the velocity is clockwise or counterclockwise.

The direction of the homogeneous acceleration $\ddot{\theta}_{H}$ required to reduce the magnitude of the joint torques can be obtained by evaluating (18). The plot in Fig. 5 graphically depicts the sign of $\ddot{\theta}_{H}$ by illustrating whether it will require a clockwise or counterclockwise acceleration around the homogeneous solution curve. This plot determines whether $\ddot{\theta}_{H}$ can be used to reduce the magnitude of the homogeneous velocity while simultaneously reducing the joint torque. Note that this is identical to the conditions guaranteeing stability for the local torque minimization scheme. If the manipulator is in a configuration where the

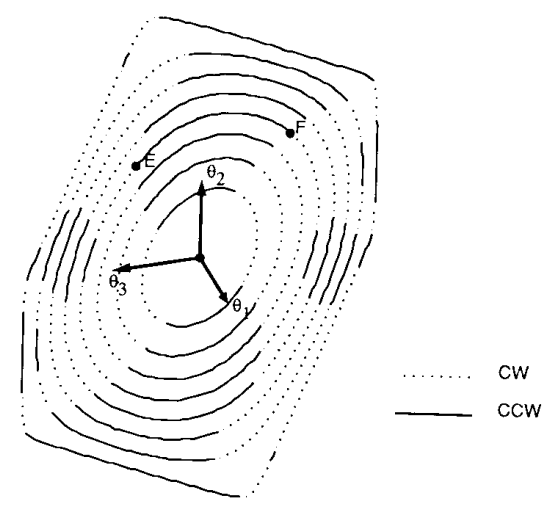

Fig. 5. A plot of the homogeneous solution curves illustrating the direction of the homogeneous acceleration required to reduce the joint torque due to a homogeneous velocity. The manipulator configuration denoted by point $E$ illustrates an inherently stable point where any homogeneous velocity can be reduced while simultaneously reducing the joint torque. Point $F$ illustrates an inherently unstable point.

homogeneous velocity is in the same direction as the acceleration given by Fig. 5 , then the local torque minimization scheme should not be applied.

The data plotted in Fig. 5 can also be used to identify possibly desirable regions of operation. In particular, those configurations that are the boundary between clockwise and counterclockwise $\ddot{\theta}_{H}$ can be classified as inherently stable or unstable depending on the direction of the transition. As an example, consider the manipulator configuration labeled $E$ in Fig. 5. Regardless of the direction of the homogeneous joint velocity, the homogeneous acceleration can always reduce this velocity while simultaneously minimizing the joint torque thus resulting in an inherently stable configuration. The manipulator configuration labeled $F$, however, is inherently unstable because, regardless of the direction of the homogeneous velocity, reducing the joint torque will always result in a homogeneous acceleration that increases the homogeneous velocity. In some respects it is useful to think of point $E$ being in a valley and point $F$ being on the top of a ridge although the analogy is not perfect.

\section{Simulation Results}

To illustrate the characteristics of stable and unstable configurations, simulations were performed in which the manipulator was put into the desired configuration, given an initial homogeneous joint velocity of $1 \mathrm{rad} / \mathrm{s}$, and then commanded to maintain a stationary end effector position. Each simulation was performed twice, once for a homogeneous velocity in the clockwise direction and then again in the counterclockwise direction. In both cases results are shown for the pseudoinverse formulation, given by (12), as a basis of comparison to the torque minimization formulation, which includes (18).

The results for the stable configuration, denoted by $E$, are presented in Fig. 6. As anticipated for this configuration, both the joint velocity norm and joint torque norm can be reduced simultaneously, regardless of the direction of the homogeneous velocity. This characteristic of stable configurations makes them ideal for decelerating a homogeneous velocity when approaching the desired homogeneous space solution for a specific secondary criterion. While the local minimum torque solution cannot in general be used to bring the homogeneous solution to zero velocity, it can be reduced to a point where the nonminimum torque solution is still well within physically achievable limits. 

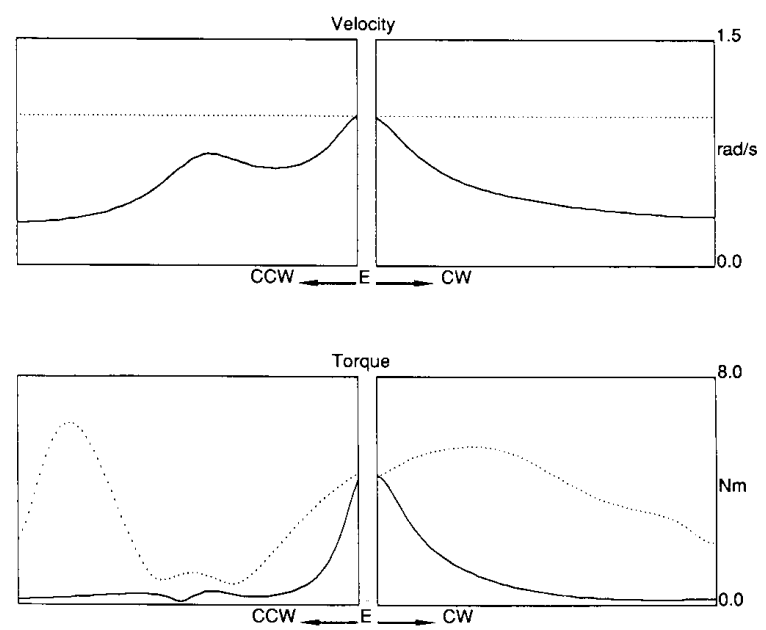

$\begin{array}{ll}\ldots . . . . & \text { Pseudoinverse } \\ & \end{array}$

Fig. 6. Simulation results showing the joint velocity norm and torque norm for the manipulator starting in an inherently stable configuration $(E)$. Simulations are performed for an initial homogeneous velocity norm of 1 $\mathrm{rad} / \mathrm{s}$, once in the clockwise direction and once in the counterclockwise direction. The results are presented for both the torque minimization formulation and the pseudoinverse formulation (no homogeneous acceleration).

For the homogeneous velocity in the counterclockwise direction there is a small hump in the velocity norm that denotes a change in sign of (20). Clearly this hump could be removed by not applying the homogeneous acceleration under these conditions as discussed above.

The results for the unstable configuration, denoted as $F$, are presented in Fig. 7. From these results one can see that the local torque minimization scheme does initially result in a decrease of the torque required to maintain the desired end effector position. However, this decrease is short lived due to the buildup of the homogeneous velocity that eventually results in physically unachievable torque requirements. It is important to note that these characteristics are not dependent on the end effector trajectory that has brought the manipulator into the unstable configuration. It should also be pointed out that these unstable configurations are not inherently undesirable. In particular, if the torque minimization formulation is only applied for limited periods of time, one can "shave" the peaks from the torque curve while waiting for a stable configuration in which to remove the induced homogeneous velocity.

There are two final points that should be addressed concerning the characteristics of homogeneous space solutions. The first is that stable configurations are in no way correlated with globally optimal minimum torque solutions. The second point relates to the fact that, unless the secondary criterion induces a large homogeneous velocity, the kinetic requirements of the primary constraint of a specified end effector trajectory will tend to dominate the dynamic behavior of the manipulator. This will usually be true until the manipulator starts to approach the end of its gross motion trajectory where the desired end effector velocity is reduced. It is at this point that consideration of the kientic effects of any residual homogeneous velocity is critical since they will tend to dominate the behavior of the manipulator.
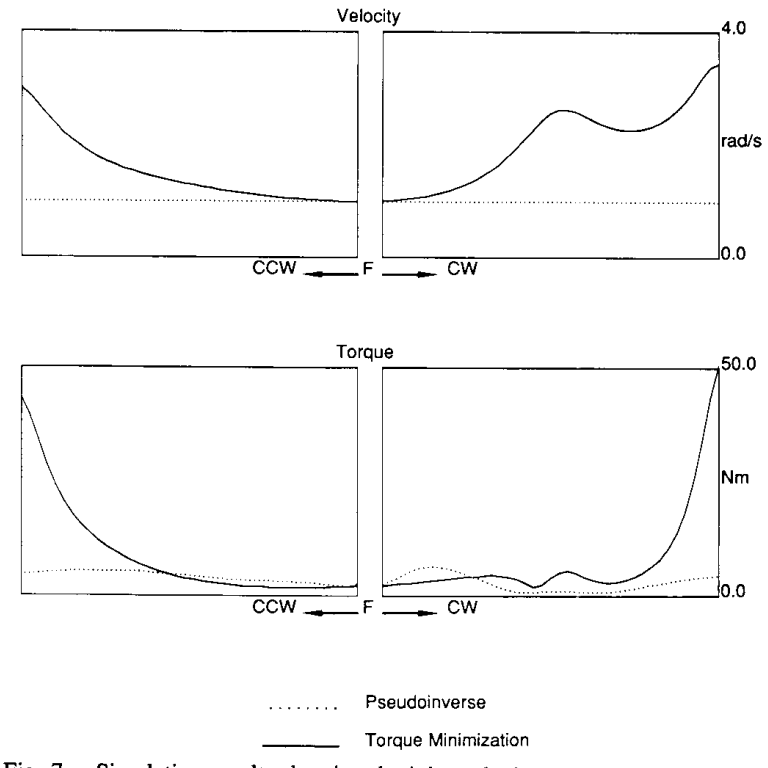

Fig. 7. Simulation results showing the joint velocity norm and torque norm for the manipulator starting in an inherently unstable configuration $(F)$. Simulations are performed for an initial homogeneous velocity norm of 1 $\mathrm{rad} / \mathrm{s}$, once in the clockwise direction and once in the counterclockwise direction. The results are presented for both the torque minimization formulation and the pseudoinverse formulation (no homogeneous acceleration).

This is particularly true of those tasks that require fine manipulation after gross motion as is typical of most assembly operations.

\section{Conclusions}

The kinematic specification of motion for redundant manipulators has relied primarily on a formulation that treats the redundant degrees of freedom as independent from those required to maintain a desired end effector trajectory. While such a formulation is conceptually appealing, it has been shown to be physically inaccurate when applied to the kinetic behavior of redundant manipulators. In this work, the kinetic effects of homogeneous solutions have been analyzed with emphasis on placing realistic limitations on how redundancy can be utilized without adversely affecting the primary goal of a desired end effector trajectory. It has been shown that it is possible to identify manipulator configurations that possess the desirable characteristic of being able to either remove or impart a homogeneous velocity while simultaneously reducing the torque requirements on the manipulator. The conditions that govern these configurations have also been shown to be directly related to the conditions for guaranteeing global stability for the local torque minimization formulation.

$$
\begin{gathered}
\text { APPENDIX } \\
\text { Simplification OF }\left[H\left(I-J^{+} J\right)\right]^{+} \text {To } \frac{v_{n} v_{n}^{T} H}{v_{n}^{T} H^{2} v_{n}}
\end{gathered}
$$

If one restricts the degree of redundancy to a single dimension, then for full row rank $J$ the projection operator can be described by the vector outer product

$$
\left(I-J^{+} J\right)=v_{n} v_{n}^{T}
$$

where $v_{n}$ is a unit vêctor in the null space of $J$. Under these 
conditions the following equality holds:

$$
\left[H\left(I-J^{+} J\right)\right]^{+}=\left[H v_{n} v_{n}^{T}\right]^{+} .
$$

It is well known [2] that for any matrix $A$ that has the full-rank factorization

$$
A=B C
$$

its pseudoinverse is given by

$$
A^{+}=C^{*}\left(B^{*} A C^{*}\right)^{-1} B^{*}
$$

where $*$ denotes the complex conjugate transpose. By substituting

$$
B=H v_{n}
$$

and

$$
C=v_{n}^{T}
$$

into (A4), one obtains

$$
\left[H v_{n} v_{n}^{T}\right]^{+}=\left(v_{n}^{T}\right)^{T}\left[\left(H v_{n}\right)^{T} H v_{n} v_{n}^{T}\left(v_{n}^{T}\right)^{T}\right]^{-1}\left(H v_{n}\right)^{T}
$$

which upon performing the transposes becomes

$$
\left[H v_{n} v_{n}^{T}\right]^{+}=v_{n}\left[v_{n}^{T} H^{T} H v_{n} v_{n}^{T} v_{n}\right]^{-1} v_{n}^{T} H^{T} .
$$

By using the fact that $v_{n}$ is a unit vector and $H$ is symmetric, this simplifies to

$$
\left[H v_{n} v_{n}^{T}\right]^{+}=\frac{v_{n} v_{n}^{T} H}{v_{n}^{T} H^{2} v_{n}}
$$

where the denominator is guaranteed to be nonzero since $H$ is strictly positive definite. Substituting this result into (A2) results in the desired equality.

\section{REFERENCES}

[1] J. Baillieul, "Kinematic programming alternatives for redundant manipulators," in Proc. 1985 IEEE Int. Conf. Robotics Automat. (St. Louis, Mar. 25-28, 1985), pp. 722-728.

[2] A. Ben-Israel and T. N. E. Greville, Generalized Inverses: Theory and Applications. New York: Krieger, 1980.

[3] B. E. Blaho, "Evaluation of dexterity measures for kinematically redundant robotic systems," M.S. thesis, Dept. of Electrical Engineering, The Ohio State University, Columbus, 1985.

[4] J. W. Burdick, " On the inverse kinematics of redundant manipulators: Characterization of the self-motion manifolds," in Proc. 1989 IEEE Int. Conf. Robotics Automat. (Scottsdale, AZ, May 14-18, 1989), pp. 264-270.

[5] P. H. Chang, "A closed-form solution for inverse kinematics of robot manipulators with redundancy," IEEE J. Robotics Automat., vol. RA-3, no. 5, pp. 393-403, Oct. 1987.

[6] S. L. Chiu, "Control of redundant manipulators for task compatibility," in Proc. 1987 IEEE Int. Conf. Robotics Automat. (Raleigh, NC, Mar.-Apr. 1987), pp. 1718-1724.

[7] R. Dubey and J. Y. S. Luh, "Redundant robot control for higher flexibility," in Proc. 1987 IEEE Int. Conf. Robotics Automat. (Raleigh, NC, Mar.-Apr. 1987), pp. 1066-1072.

[8] J. M. Hollerbach and K. C. Suh, "Redundancy resolution of manipulators through torque optimization," IEEE J.Robotics Automat., vol. RA-3, no. 4, pp. 308-316, Aug. 1987.

[9] C. A. Klein, "Use of redundancy in the design of robotic systems," in Robotics Research: The Second International Symposium, H. Hanafusa and H. Inoue, Eds. Cambridge, MA: MIT Press, 1985 pp. 207-214.

[10] C. A. Klein and B. E. Blaho, "Dexterity measures for the design and control of kinematically redundant manipulators," Int. J. Robotics Res., vol. 6, no. 2, pp. 72-83, Summer 1987.

[11] C. A. Klein and A. I. Chirco, "Dynamic simulation of a kinematically redundant manipulator system," J. Robotic Systems, vol. 4, no. 1, pp. 5-23, 1987.

[12] C. A. Klein and C. H. Huang, "Review of pseudoinverse control for use with kinematically redundant manipulators," IEEE Trans. Syst., Man Cyber., vol. SMC-13, pp. 245-250, Mar./Apr. 1983.

[13] A. Liegeois, "Automatic supervisory control of the configuration and behavior of multibody mechanisms," IEEE Trans. Syst. Man Cyber., vol. SMC-7, no. 12, pp. 868-871, Dec. 1977.

[14] A. A. Maciejewski and C. A. Klein, "Obstacle avoidance for kinematically redundant manipulators in dynamically varying environments," Int. J. Robotics Res., vol. 4, no. 3, pp. 109-117, Fall 1985.

[15] A. A. Maciejewski, "The analysis of kinematically redundant manipulators through computer graphic simulation," in Proc. 20th Ann. Pittsburgh Conf. Modeling and Simulation (Pittsburgh, PA, May 4-5, 1989).

[16] R. V. Mayorga and A. K. C. Wong, "A singularities avoidance approach for the optimal local path generation of redundant manipulators," in Proc. 1988 IEEE Int. Conf. Robotics Automat. (Philadelphia, Apr. 24-29, 1988), pp. 49-54.

[17] Y. Nakamura, H. Hanafusa, and T. Yoshikawa, "Task-priority based redundancy control of robot manipulators," Int. J. Robotics Res., vol. 6, no. 2, pp. 3-15, Summer 1987.

[18] T. Yoshikawa, "Analysis and control of robot manipulators with redundancy," in Robotics Research: The First International Symposium, M. Brady and R. Paul, Eds. Cambridge, MA: MIT Press, 1984, pp. 735-747.

[19] - "Manipulability of robotic mechanisms," Int. J. Robotics Res., vol. 4, no. 2, pp. 3-9, Summer 1985.

[20] -, "Dynamic manipulability of robot manipulators," $J$. Robotic Syst., vol. 2, no. 1, pp. 113-124, 1985.

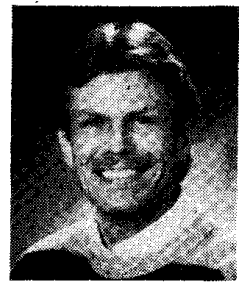

Anthony A. Maciejewski (' '82-M'87) received the B.S.E.E., M.S., and Ph.D. degrees in electrical engineering from The Ohio State University, Columbus, in 1982, 1984, and 1987, respectively.

Since 1988, has been an Assistant Professor with the School of Electrical Engineering at Purdue University, West Lafayette, IN. His primary research interests center on the simulation and control of kinematically redundant systems. 